\title{
BMJ Open Association between long-term oxygen therapy provided outside the guidelines and mortality in patients with COPD
}

\author{
Francois Alexandre (D) , ${ }^{1}$ Virginie Molinier, ${ }^{1}$ Maurice Hayot, ${ }^{2}$ Guillaume Chevance, ${ }^{3}$ \\ Gregory Moullec, ${ }^{4}$ Alain Varray (D) ${ }^{5}$ Nelly Héraud ${ }^{1}$
}

To cite: Alexandre F, Molinier V, Hayot M, et al. Association between long-term oxygen therapy provided outside the guidelines and mortality in patients with COPD. BMJ Open 2022;12:e049115. doi:10.1136/ bmjopen-2021-049115

- Prepublication history and additional supplemental material for this paper are available online. To view these files, please visit the journal online (http://dx.doi.org/10.1136/ bmjopen-2021-049115).

FA and VM contributed equally.

FA and VM are joint first authors.

Received 18 January 2021 Accepted 01 December 2021

A) Check for updates

(c) Author(s) (or their employer(s)) 2022. Re-use permitted under CC BY-NC. No commercial re-use. See rights and permissions. Published by BMJ.

For numbered affiliations see end of article.

\section{Correspondence to}

Mr Francois Alexandre; alexandre.francois88@gmail. com

\section{ABSTRACT}

Introduction Hypoxaemia is a frequent complication of chronic obstructive pulmonary disease (COPD). To prevent its consequences, supplemental oxygen therapy is recommended by international respiratory societies. However, despite clear recommendations, some patients receive long-term oxygen therapy (LTOT), while they do not meet prescription criteria. While evidence suggests that acute oxygen supply at high oxygenation targets increases COPD mortality, its chronic effects on COPD mortality remain unclear. Thus, the study will aim to evaluate through a systematic review and individual patient data meta-analysis (IPD-MA), the association of LTOT prescription outside the guidelines on survival over time in COPD.

Methods Systematic review and IPD-MA will be conducted according to Preferred Reporting Items for a Systematic Review and Meta-Analyses IPD guidelines. Electronic databases (PubMed, Web of Science, EMBASE, Cochrane Central Register of Controlled Trials, ClinicalTrials.gov, OpenGrey and BioRxiv/MedRxix) will be scanned to identify relevant studies (cohort of stable COPD with arterial oxygen tension data available, with indication of LTOT filled out at the moment of the study and with a survival follow-up). The anticipated search dates are January-February 2022. The main outcome will be the association between LTOT and time to all-cause mortality according to hypoxaemia severity, after controlling for potential covariates and all available clinical characteristics. Quantitative data at the level of the individual patient will be used in a one-step approach to develop and validate a prognostic model with a Cox regression analysis. The one-step IPD-MA will be conducted to study the association and the moderators of association between supplemental oxygen therapy and mortality. Multilevel survival analyses using Cox-mixed effects models will be performed. Ethics and dissemination As a protocol for a systematic review, a formal ethics committee review is not required. Only studies with institutional approval from an ethics committee and anonymised IPD will be included. Results will be disseminated through peer-reviewed publications and presentations in conferences.

PROSPERO registration number CRD42020209823.

\section{INTRODUCTION}

Chronic obstructive pulmonary disease (COPD) is a common respiratory illness involving near to $9 \%$ of adult population and becoming the third cause of death in the world. ${ }^{12}$ This disease leads, among others,

\section{Strengths and limitations of this study}

- Systematic review will provide the most important dataset available on patients with long-term oxygen therapy (LTOT) prescription outside current guidelines

- The individual patient data (IPD) meta-analysis will offer new evidences concerning the association between LTOT provided outside the guidelines and mortality hazard rates.

- The observational data collected in this study will be closer to real-world data than in previous randomized controlled trials.

- Limitation includes the impossibility to directly conclude on the existence of a potential cause-effect relationship between LTOT and mortality.

- Limitation also lies on the ability to acquire the IPD and on the evaluation of the heterogeneity of the study designs and studied populations.

to respiratory problems, pulmonary hypertension, heart failure, neurocognitive and neuromuscular dysfunctions and systemic inflammation. ${ }^{3}$ Directly linked to the respiratory complications, patients with COPD usually suffer from hypoxaemia issues (transient when limited to sleep or exercise or chronic when also occurring at rest). The degree of hypoxaemia worsens with the progression of the disease up to respiratory insufficiency. Hypoxaemia is classically defined as an abnormally arterial oxygen tension level $\left(\mathrm{PaO}_{2}\right)$ lower than $80 \mathrm{~mm} \mathrm{Hg}$ or an arterial oxygen saturation $\left(\mathrm{SaO}_{2}\right.$ or $\mathrm{SpO}_{2}$ ) lower than $93 \%$ at rest while breathing ambient air. ${ }^{45}$ In COPD, severe hypoxaemia is defined as $\mathrm{PaO}_{2}$ breathing room air at rest less than $55 \mathrm{~mm} \mathrm{Hg}$ or less than $59 \mathrm{~mm} \mathrm{Hg}$ with concurrent polycythaemia or signs of right-sided heart failure or pulmonary hypertension. Therefore, non-severe hypoxaemia (ie, mild to moderate) is seen as a $\mathrm{PaO}_{2}$ higher than $59 \mathrm{~mm} \mathrm{Hg}$ but less than normal value $(80 \mathrm{~mm} \mathrm{Hg}$ in general or less according to age $\left.{ }^{67}\right)$. 
Hypoxaemia is considered as a major complication of COPD, notably associated with decreased exercise tolerance $^{8}$ and increased mortality. ${ }^{9}$ To prevent its consequences, supplemental oxygen therapy is usually prescribed either in case of transient hypoxaemia during exercise or/and sleep, or in case of chronic hypoxaemia using a long-term oxygen therapy (LTOT) device (ie, prescription of supplemental oxygen therapy at least 15 hours per day). LTOT has been recommended for decades by international guidelines for severe hypoxaemic patients exclusively. ${ }^{1011}$ These recommendations are based on two landmark studies published in early 1980s, which are the sole to date to have demonstrated some substantial benefits of providing LTOT in COPD. Briefly, the Medical Research Council study reported higher survival rate of 5 years after randomisation in severe hypoxaemic patients receiving supplemental oxygen therapy for greater than 15 hours per day versus no oxygen at all. ${ }^{12}$ In addition, the Nocturnal Oxygen Therapy Trial study found higher survival rate of 3 years after randomisation in severe hypoxaemic patients receiving continuous supplemental oxygen therapy versus those receiving 12 hours nocturnal supplemental oxygen therapy. ${ }^{13}$

Although the last international guidelines recommend to restrain LTOT to severe hypoxaemic patients, ${ }^{14} 15$ several studies reported a large proportion of patients under LTOT (around 40\%) while having only mild-tomoderate hypoxaemia. ${ }^{16-20}$ These prescriptions of LTOT outside current guidelines (ie, supplemental oxygen therapy prescribed at least 15 hours per day in patients with no severe hypoxaemia) can be explained by at least two reasons. First, LTOT is most of the time prescribed after an exacerbation, thus in an instability context, and without further reevaluation of the patient's need. ${ }^{21} 22$ However, hypoxaemia status is variable and some patients may recover over time. For instance, it has been shown that up to $40 \%$ patients who meet criteria for LTOT prescription at a given time have spontaneously recovered $1-3$ months later. ${ }^{18}$ Second, LTOT is sometimes prescribed outside the guidelines for prevention or convenience purposes. Indeed, almost $20 \%$ of patients are prescribed LTOT while not having severe hypoxaemia even at the time of prescription. ${ }^{23}{ }^{24}$ This lack of adherence to LTOT prescription guidelines could be linked to the embedded culture of 'more is better' and 'it's just oxygen, it doesn't do any harm', which has been highlighted in a recent qualitative survey on the myths and beliefs regarding oxygen therapy prescription. ${ }^{19}$

In addition to the considerable economic costs generated, the health consequences of LTOT provided outside the guidelines are an important issue by unnecessarily exposing patients to the potential adverse effects of hyperoxia. These effects have already been documented under acute exposition. Indeed, a randomized controlled trial (RCT) on patients with acute exacerbated COPD found that high flow oxygen therapy (hyperoxaemia) in the pre-hospital setting, compared with titrated supplemental oxygen therapy (normoxaemia) induced an almost five-time increase of mortality. ${ }^{25}$ Furthermore, an additional retrospective study on patients with exacerbated COPD admitted in intensive care unit found that hyperoxaemia, that had occurred in $24 \%$ of patients, was associated with an increased risk of serious inpatient adverse outcomes (death, assisted ventilation or respiratory failure) $(\mathrm{OR}=9.17,95 \% \mathrm{CI} 4.08$ to 20.60$) .{ }^{26}$ Altogether, these data show that supplemental oxygen therapy at high oxygenation targets increases COPD mortality in the acute settings.

However, while the potential adverse effects of acute oxygen supply in COPD have been established, those induced by chronic exposition are inconsistent. The impact of LTOT in mild-to-moderate hypoxaemic patients with COPD has been assessed in two RCTs, both showing no effect on mortality risk. ${ }^{27} 28$ However, they both have important limitations that impede any definitive conclusion. First, the study of Górecka et $a l^{27}$ recorded too small numbers of deaths to conclude with certainty on the absence of mortality rate differences between groups (only 38 and 32 non-survivors in the LTOT and control group, respectively). Furthermore, the Long-term Oxygen Treatment Trial (LOTT) ${ }^{28}$ only indirectly estimated hypoxaemia severity through pulsed oxygen saturation $\left(\mathrm{SpO}_{2}\right)$. While the authors targeted to enrol patients with an $\mathrm{SaO}_{2}$ between $89 \%$ and $93 \%,{ }^{28}$ which is thought to be equivalent to a $\mathrm{PaO}_{2}$ of $59-70 \mathrm{~mm}$ $\mathrm{Hg}, \mathrm{SpO}_{2}$ is known to give a mean $\mathrm{SaO}_{2}$ overestimation of 4 points. ${ }^{29}$ Thus, with a mean $\mathrm{SpO}_{2}$ of $92 \%$, it is possible that some patients had severe hypoxaemia instead of mild-to-moderate one in the LOTT study. ${ }^{28}$ Besides those RCTs, a few retrospective observational studies also aimed to assess the association between mortality and LTOT prescription outside the guidelines. ${ }^{172030}$ On one hand, Drummond $e t a l^{17}$ found an association between mortality and LTOT outside the guidelines, but it was not significant anymore after controlling by confounding factors. On the other hand, Alexandre et $a l^{30}$ and Pavlov et $a l^{20}$ reported a strong tendency toward increased mortality in patients under LTOT, but the samples were too small to definitely conclude.

The impact of LTOT prescription outside the guidelines on mortality risk thus needs to be clarified. Conducting a specific RCT would be costly and challenging. In this context, the completion of a systematic review and meta-analysis remains a relevant alternative. Although very few studies aimed to specifically address the association between LTOT outside the guidelines and mortality in COPD, re-analysis of available datasets is a possible option. Indeed, in the literature, $\mathrm{PaO}_{2}$ data and oxygen therapy status are almost systematically reported for each patient to characterise the severity of COPD. In the studies which also assessed mortality, it will be possible to carry out re-analyses in order to verify whether the mortality rates evolve differently according to the association of $\mathrm{PaO}_{2}$ and LTOT. Individual participant data meta-analysis (IPD-MA) is a reliable and relevant tool to address this question in the present context. Performing 
an IPD-MA requires synthesis of patient-level data across multiple studies. Those kind of meta-analyses are considered as gold-standard for several reasons: (1) they allow greater statistical flexibility to control for individual variability compared with aggregate meta-analysis, which can only account for variance at the study level, ${ }^{31}$ (2) statistical analyses can be adapted to consistently accommodate the features of the data from each study and differences in study setting (inclusion/exclusion criteria, missing data, differences for measuring effect size, time effect, etc) and (3) potential statistical bias or poorly reported outcomes from individual study can be verified and modelled in a more appropriate way (see Riley $e t a{ }^{32}$ for further advantages).

\section{OBJECTIVES OF THE IPD-MA}

This project will address this specific question: does LTOT provided outside the guidelines (ie, in patients without severe hypoxaemia) increase the HR of death in patients with COPD? Given the reported adverse effects of supplemental oxygen therapy at high oxygenation targets in the acute settings, we hypothesise that patients with COPD chronically exposed to supplemental oxygen therapy at high oxygenation targets (ie, patients who are prescribed LTOT outside the guidelines) have a higher HR of death compared with patients with COPD under LTOT in line with the guidelines or all patients with COPD without LTOT (outside or in line with the guidelines).

To address the question, relevant studies combining a measure of hypoxaemia degree, survival status and LTOT status among patients with COPD will be identified through a systematic review. Then, the datasets of selected studies will be analysed to compare the association between LTOT and mortality in patients with COPD as a function of hypoxaemia severity.

\section{METHODS AND ANALYSES \\ Patient and public involvement \\ No patient involved.}

\section{Registration}

The protocol was designed in accordance with the PRISMA-IPD guidelines ${ }^{33}$ and is registered on the PROSPERO database.

\section{Eligibility criteria for selected studies \\ Types of studies}

All retrospective or prospective cohort studies from any countries and published in English or French languages with no date restriction will be included. Databases from unpublished studies and not yet published studies but meeting inclusion criteria might be included in the study. Thus, by openly publishing this protocol, the authors also invite all readers with such databases to share them in order to contribute to the project. In addition, the protocol will be shared with specific research networks working on the study topic.

\section{Population}

The subjects of the selected studies will have to be older than 40 years with a COPD diagnosis, defined as a documented forced expiratory volume in the first second of forced expiration $\left(\mathrm{FEV}_{1}\right)$ /forced vital capacity (FVC) ratio $<0.70$ after bronchodilator intake. In addition, the studies will have to verify the following four conditions:

- Cohort of stable COPD (last exacerbation $>4$ weeks) at the moment of the study.

- Partial arterial oxygen values data during breathing ambient air in resting conditions available

- Indication of the presence or absence of LTOT (ie, prescription of at least 15 hours/day) filled out at the moment of the study.

- Survival follow-up, without any time restriction on the follow-up duration

\section{Exclusion criteria}

Cohorts with specific selection of subgroup of patients based on hypoxaemia severity (eg, studies only focusing on severe hypoxaemia and studies including only patients with normoxaemic COPD) will not be included.

\section{Interventions/exposure and comparator groups}

The exposure group will be stable patients with COPD with LTOT despite not meeting the LTOT prescription criteria (ie, patients with a $\mathrm{PaO}_{2}$ higher than $59 \mathrm{~mm} \mathrm{Hg}$ at the time of the prescription or/and at the time of inclusion in the study).

Three comparator groups will be investigated:

- Stable patients with COPD with LTOT prescription who meet the LTOT prescription criteria (ie, $\mathrm{PaO}_{2}$ less than $55 \mathrm{~mm} \mathrm{Hg}$ or less than $59 \mathrm{~mm} \mathrm{Hg}$ with concurrent polycythaemia or signs of right-sided heart failure or pulmonary hypertension),

- Stable patients with COPD without LTOT prescription and who do not meet the prescription criteria (ie, $\mathrm{PaO}_{2}$ higher than $59 \mathrm{~mm} \mathrm{Hg}$ or $\mathrm{PaO}_{2}$ between $55 \mathrm{~mm} \mathrm{Hg}$ and $59 \mathrm{~mm} \mathrm{Hg}$ without concurrent polycythaemia or signs of right-sided heart failure or pulmonary hypertension),

- Stable patients with COPD without LTOT prescription despite meeting the LTOT prescription criteria (ie, $\mathrm{PaO}_{2}$ less than $55 \mathrm{~mm} \mathrm{Hg}$ or less than $59 \mathrm{~mm} \mathrm{Hg}$ with concurrent polycythaemia or signs of right-sided heart failure or pulmonary hypertension).

Types of outcome measures

We will include studies that report the time to death (allcause mortality).

\section{Exclusion criteria}

We will exclude book chapters and systematic reviews (but reference lists will be checked as mentioned above).

\section{PICOS summary}

The criteria for including studies in the review can be as summarised in table 1. 


\begin{tabular}{|c|c|c|c|c|}
\hline Population & Intervention/exposure & Comparator & Outcomes & Setting \\
\hline $\begin{array}{l}\text { Patients with COPD ( }>40 \text { years old). } \\
\text { In addition, the studies will have to verify the } \\
\text { following } 4 \text { conditions: } \\
\text { Cohort of stable COPD (last exacerbation }>4 \\
\text { weeks) at the moment of the study. } \\
\text { Partial arterial oxygen values data during } \\
\text { breathing ambient air in resting conditions } \\
\text { available. } \\
\text { Indication of the presence or absence of LTOT (ie, } \\
\text { prescribed at least } 15 \text { hours/day) filled out at the } \\
\text { moment of the study. } \\
\text { Survival follow-up for at least } 12 \text { months after the } \\
\text { inclusion in the study }\end{array}$ & Patients with LTOT & $\begin{array}{l}\text { Patients } \\
\text { without LTOT }\end{array}$ & $\begin{array}{l}\text { Mortality } \\
\text { (time to } \\
\text { death) }\end{array}$ & $\begin{array}{l}\text { Retrospective } \\
\text { cohort studies and } \\
\text { prospective cohort } \\
\text { studies }\end{array}$ \\
\hline
\end{tabular}

COPD, chronic obstuctive pulmonary disease; LTOT, long-term oxygen therapy.

\section{Study outcomes}

The main outcome for the IPD-MA will be time to death. The time to death will be calculated as the number of days between the date of inclusion in the selected study or, if available, from oxygen prescription (index date) to death or end date of studies, which ever came first. The survival times of patients still alive will be censored at the date of last follow-up.

\section{Moderator analyses}

Characteristics of the patients (patient-level moderators)

The following covariates known to influence mortality in COPD will be integrated in the analyses: age, sex, body mass index (BMI), arterial carbon dioxide tension $\left(\mathrm{PaCO}_{2}\right)$, exercise tolerance (6-minute walking distance), dyspnoea (modified Medical Research Council (mMRC) score), comorbidities (COPD-specific co-morbidity test (COTE) index), $\mathrm{FEV}_{1}$ and the presence of at least one of the following three conditions: polycythaemia or signs of right-sided heart failure or pulmonary hypertension.

\section{Characteristics of the studies (study-level moderators)}

Time: timing of outcome and time of follow-up will also be assessed and will be considered for mortality analyses since timeframes will be different between studies. An important source of heterogeneity is length of follow-up as this may impact the hazard of death. These aspects will be carefully detailed during the review process and controlled for in our analyses (eg, by running a moderator analysis with the length of follow-up as a continuous variable or, if few variability is found for this outcome, by performing subgroup analyses for each length of follow-up).

\section{Data collection}

Search strategy and methods for the identification of studies The protocol was prepared according to the guidelines of the Preferred Reporting Items for a Systematic Review and Meta-Analyses of IPD (PRISMA-IPD) Development Group. ${ }^{33}$ A multistage search strategy will be completed to identify all potential datasets (figure 1). Literature search strategies will be developed using text words for all databases or using medical subject headings (MeSH for MEDLINE) related to COPD-LTOT-survival-follow-up study. MEDLINE (PubMed platform), Web of Science, EMBASE and Cochrane Central Register of Controlled Trials bibliographic databases will be searched from database inception to the present day. ClinicalTrial registry will also be searched to identify any unpublished trial. Authors of these trials will be contacted via email and invited to share unpublished data. Databases with unpublished data will be checked and details (eg, origin, context of data collection, etc) will be then specified. Finally, articles from grey literature and from preprint will be searched on OpenGrey platform (http://www.opengrey.eu/) and BioRxiv/MedRxix platforms (https://www.biorxiv. org and https://www.medrxiv.org, respectively). Bibliography of eligible articles as well as existing systematic reviews in the field will also be screened. Extra studies or datasets not found after databases searching but meeting inclusion criteria will be included through a snowballing search. Subsequently, the authors of eligible studies will be contacted and asked to share IPD.

Screening, study selection and electronic database of the study For each database, registry and resources platform, searching will be conducted using different strategy steps and different combinations of searching terms (see online supplemental appendix 1).

Study selection will be performed by two authors (FA and VM). They will independently screen the titles and abstracts of retrieved studies identified by the search strategies and if these meet inclusion criteria. Following the initial identification and removal of duplicates, these potentially eligible studies will be re-evaluated in the full text by two authors (FA and VM) for inclusion criteria checking defined previously. Disagreements will be resolved through discussion and if not, a third author (NH) will decide. 


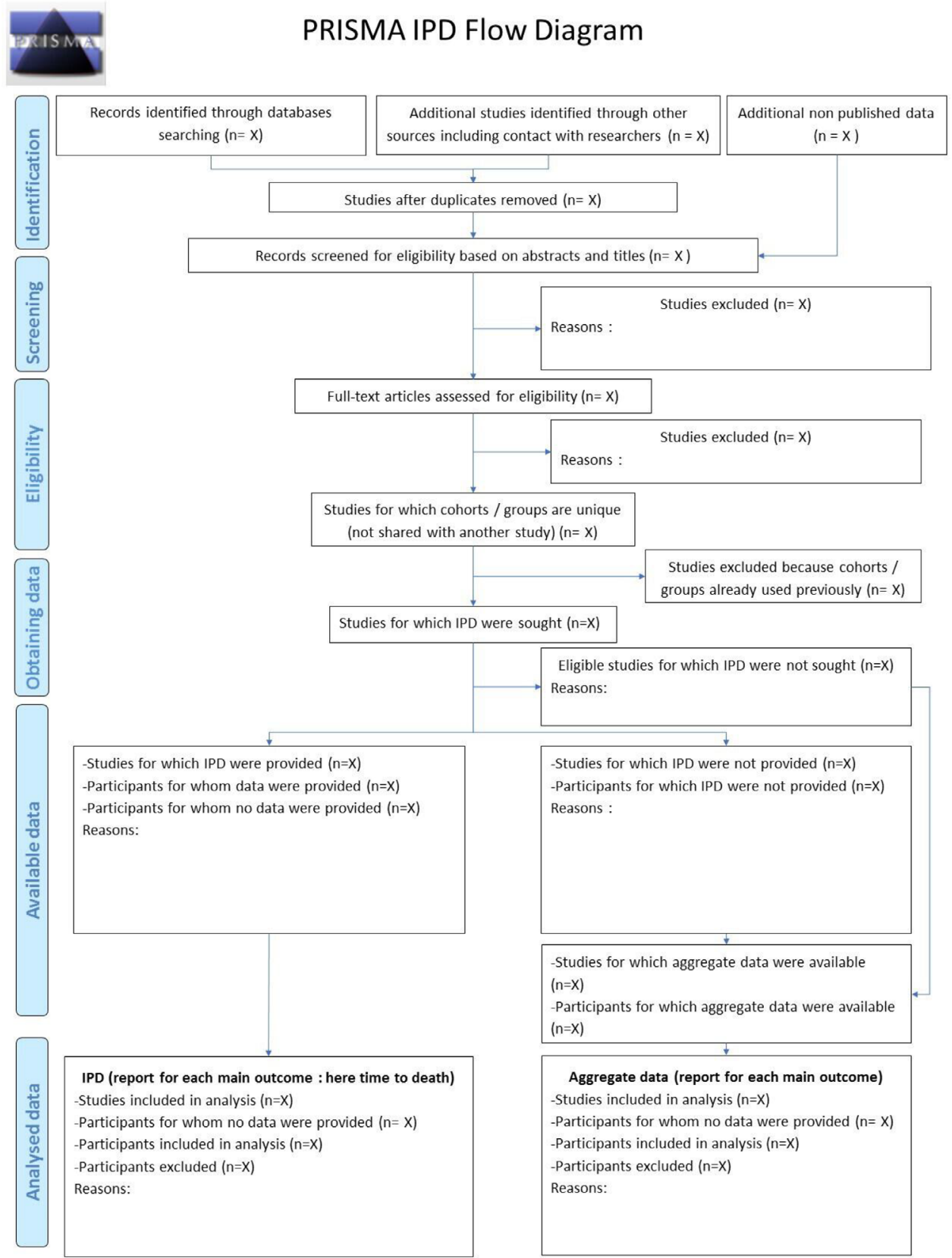

Figure 1 PRISMA-IPD flow diagram. PRISMA-IPD, Preferred Reporting Items for a Systematic Review and Meta-Analyses of Individual Participant Data.

\section{Data extraction and management}

Study selection procedure will be presented in a PRISMA-IPD flow diagram (figure 1). The final results from the literature search will be downloaded and saved into the Zotero reference management software. Data will be extracted onto a datasheet by one author and later checked for accuracy by a second author. Extracted data will include study characteristics (title, corresponding authors' contact details, publication year, journal and type of study), population characteristics (number of patients with COPD, age and sex ratio), characteristics of the intervention and comparator and the different sought outcomes. Once the studies will be selected and extracted, corresponding authors of the identified eligible published studies and trials will be contacted via email with a cover letter, or in a personal conversation, detailing the objectives of the collaborative meta-analysis, background information and the datasheet for input of individual patient results for the project. Indeed, those responsible for the included studies will be asked to supply individual participant data comprising de-identified participants reference. Moreover, owners of unpublished selected databases will be contacted in the same manner.

A master database will be created grouping all respective datasheets and each study will have an ID number. If some IPD characteristics or outcomes are missing, unclear or not existent in the dataset provided, we will 
Table 2 Individual data requested to authors for IPD-MA

\section{Patient-level data}

\begin{tabular}{|c|c|c|c|}
\hline Study parameters & Patient details & Intervention/treatment & Outcomes \\
\hline Follow-up time period & 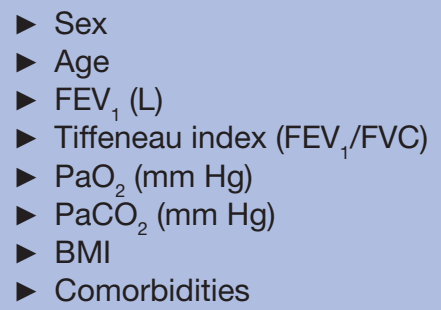 & $\begin{array}{l}\text { LTOT (ie, prescribed at least } 15 \\
\text { hours/day) at the inclusion time }\end{array}$ & $\begin{array}{l}\text { Vital status at publication } \\
\text { time } \\
- \text { Death date }\end{array}$ \\
\hline
\end{tabular}

\section{Study-level data}

\section{- Country of completion}

- Date of publication

- Methodological quality of study

Reasons for missing data

$\mathrm{BMI}$, body mass index; $\mathrm{FEV}_{1}$, forced expiratory volume in one second; FVC, forced vital capacity; IPD-MA, individual patient data metaanalysis; LTOT, long-term oxygen therapy; $\mathrm{PaCO}_{2}$, arterial carbon dioxide tension; $\mathrm{PaO}_{2}$, arterial oxygen tension.

report them in study limitations. All IPD will be saved on a secure server. IPD integrity: individual datasets will be checked for missing, invalid, out of range or inconsistent items.

\section{Risk of bias and quality assessment}

Risk of bias and quality assessment will be carried out independently by two researchers for each included study using the Newcastle Ottawa Scale (NOS) for cohort studies $^{34}$ (see online supplemental appendix 2). The NOS contains eight questions allowing to assess information bias, selection bias and confounding in cohort studies. Study will be graded as being of good, fair or poor quality. ${ }^{35}$ Disagreements will be resolved by discussion between the two reviewers. If a risk of bias for a study remains unclear, corresponding authors of each study could be asked to provide additional information to resolve the uncertainty.

\section{Individual participant data meta-analysis \\ Data items}

The different data types which will be requested are mentioned in table 2.

\section{Statistical analyses}

The conduct and reporting of this IPD-MA will rely on the PRISMA-IPD statement ${ }^{33}$ and following the recommendations of a specific statistical tutorial for this kind of analyses. ${ }^{36}$ A one-step IPD-MA will be conducted to study the association between oxygen therapy and hazard of death using multilevel survival analyses using Cox-mixed effects models with two levels structure (patient and study) will be performed. Subgroup analyses will be run to identify the role of each potential moderators described above (ie, age, gender, $\mathrm{BMI}, \mathrm{PaCO}_{2}$, comorbidities, $\mathrm{FEV}_{1}$ and the presence of at least one of the following condition: polycythaemia or signs of right-sided heart failure or pulmonary hypertension). Missing data will be described but no imputation will be performed, as it is recommended for that kind of analysis. ${ }^{37}$ According to the method described in Austin, ${ }^{36}$ the following R packages will be used: survival, lme 4 and coxme. All statistical analyses will be independently replicated by one of the co-authors and an external collaborator. Data and codes will be made available online on medRxiv Data Repository at the time of the submission.

\section{ETHICS AND DISSEMINATION}

This paper contains the original study protocol. Any substantial modifications to the protocol will be listed and justified in a supplementary document according to the PRISMA Protocols 2015 guidelines. These amendments will be documented in detail in the PROSPERO Register and will be described transparently. Only studies that have received institutional approval from an ethics committee and present anonymised IPD will be included in the meta-analysis. As this is a protocol for a systematic review, an ethics committee review is not required. A data sharing agreement will be signed between the authors of included studies and the IPD-MA research team. The resulting IPD-MA dataset will be shared and made publicly available through the medRxiv Data Repository (accessed via https://www.medrxiv.org/) in accordance with open-science practices. Results will be disseminated via scientific forums, including peer-reviewed publications and presentations at international conferences.

\section{Author affiliations}

${ }^{1}$ Direction de la recherche clinique et de l'innovation en santé, Korian SA, Lodève, France

${ }^{2}$ PhyMedExp, Université de Montpellier, INSERM, CNRS, CHRU de Montpellier, Montpellier, France

${ }^{3}$ Barcelona Institute for Global Health, Barcelona, Spain 
${ }^{4}$ Department of Social and Preventive Medicine, School of Public Health, University of Montreal, Montreal, Quebec, Canada

${ }^{5}$ EuroMov Digital Health in Motion, IMT Mines Ales, University of Montpellier, Montpellier, France

Contributors VM is the guarantor of the review. FA, NH and VM designed the project. FA, GC, MH, NH, VM, GM and AV contributed to study methods, writing the paper, read and approved the final version of the manuscript for submission.

Funding The authors have not declared a specific grant for this research from any funding agency in the public, commercial or not-for-profit sectors.

\section{Competing interests None declared.}

Patient consent for publication Not applicable.

Provenance and peer review Not commissioned; externally peer reviewed.

Data availability statement No data are available. There is no data available related to this protocol.

Supplemental material This content has been supplied by the author(s). It has not been vetted by BMJ Publishing Group Limited (BMJ) and may not have been peer-reviewed. Any opinions or recommendations discussed are solely those of the author(s) and are not endorsed by BMJ. BMJ disclaims all liability and responsibility arising from any reliance placed on the content. Where the content includes any translated material, BMJ does not warrant the accuracy and reliability of the translations (including but not limited to local regulations, clinical guidelines, terminology, drug names and drug dosages), and is not responsible for any error and/or omissions arising from translation and adaptation or otherwise.

Open access This is an open access article distributed in accordance with the Creative Commons Attribution Non Commercial (CC BY-NC 4.0) license, which permits others to distribute, remix, adapt, build upon this work non-commercially, and license their derivative works on different terms, provided the original work is properly cited, appropriate credit is given, any changes made indicated, and the use is non-commercial. See: http://creativecommons.org/licenses/by-nc/4.0/.

\section{ORCID iDs}

Francois Alexandre http://orcid.org/0000-0003-4453-3004

Alain Varray http://orcid.org/0000-0003-3876-1211

\section{REFERENCES}

1 Roth GA, Abate D, Abate KH, et al. Global, regional, and national age-sex-specific mortality for 282 causes of death in 195 countries and territories, 1980-2017: a systematic analysis for the global burden of disease study 2017. Lancet 2018;392:1736-88.

2 Lozano R, Naghavi M, Foreman K, et al. Global and regional mortality from 235 causes of death for 20 age groups in 1990 and 2010: a systematic analysis for the global burden of disease study 2010. Lancet 2012;380:2095-128.

3 Barnes PJ, Celli BR. Systemic manifestations and comorbidities of COPD. Eur Respir J 2009;33:1165-85.

4 Mellemgaard K. The alveolar-arterial oxygen difference: its size and components in normal man. Acta Physiol Scand 1966;67:10-20.

5 Sorbini CA, Grassi V, Solinas E, et al. Arterial oxygen tension in relation to age in healthy subjects. Respiration 1968;25:3-13.

6 Cerveri I, Zoia MC, Fanfulla F, et al. Reference values of arterial oxygen tension in the middle-aged and elderly. Am J Respir Crit Care Med 1995; 152:934-41.

7 Hardie JA, Vollmer WM, Buist AS, et al. Reference values for arterial blood gases in the elderly. Chest 2004;125:2053-60.

8 Antonucci R, Berton E, Huertas A, et al. Exercise physiology in COPD. Monaldi Arch Chest Dis 2003:59:134-9.

9 Zielinski J, MacNee W, Wedzicha J, et al. Causes of death in patients with COPD and chronic respiratory failure. Monaldi Arch Chest Dis 1997;52:43-7.

102019 Global Strategy for prevention, diagnosis and management of COPD 2018.

11 Qaseem A, Wilt TJ, Weinberger SE, et al. Diagnosis and management of stable chronic obstructive pulmonary disease: a clinical practice guideline update from the American College of physicians, American College of chest physicians, American thoracic Society, and European respiratory Society. Ann Intern Med 2011;155:179-91.
12 Party MW. Long-term domiciliary oxygen therapy in chronic hypoxic cor pulmonale complicating chronic bronchitis. Lancet 1981;1:681-5.

13 Continuous or nocturnal oxygen therapy in hypoxemic chronic obstructive lung disease: a clinical trial. nocturnal oxygen therapy trial group. Ann Intern Med 1980;93:391-8.

14 Hardinge M, Annandale J, Bourne S, et al. British thoracic Society guidelines for home oxygen use in adults. Thorax 2015;70:i1-43.

15 Jacobs SS, Krishnan JA, Lederer DJ, et al. Home oxygen therapy for adults with chronic lung disease. An official American thoracic Society clinical practice guideline. Am J Respir Crit Care Med 2020;202:e121-41

16 Casaburi R, Porszasz J, Hecht A, et al. Influence of lightweight ambulatory oxygen on oxygen use and activity patterns of COPD patients receiving long-term oxygen therapy. COPD 2012;9:3-11.

17 Drummond MB, Blackford AL, Benditt JO, et al. Continuous oxygen use in nonhypoxemic emphysema patients identifies a high-risk subset of patients: retrospective analysis of the National emphysema treatment trial. Chest 2008;134:497-506.

18 Guyatt GH, Nonoyama M, Lacchetti C, et al. A randomized trial of strategies for assessing eligibility for long-term domiciliary oxygen therapy. Am J Respir Crit Care Med 2005;172:573-80.

19 Kelly CA, Lynes D, O'Brien MR, et al. A wolf in sheep's clothing? Patients' and healthcare professionals' perceptions of oxygen therapy: An interpretative phenomenological analysis. Clin Respir J 2018;12:616-32.

20 Pavlov N, Haynes AG, Stucki A, et al. Long-term oxygen therapy in COPD patients: population-based cohort study on mortality. Int $J$ Chron Obstruct Pulmon Dis 2018;13:979-88.

21 Ekström M, Ringbaek T. Which patients with moderate hypoxemia benefit from long-term oxygen therapy? Ways forward. Int J Chron Obstruct Pulmon Dis 2018;13:231-5.

22 Güell Rous R. Long-term oxygen therapy: are we prescribing appropriately? Int J Chron Obstruct Pulmon Dis 2008;3:231-7.

23 Melloni B, Mounier L, Laaban J-P, et al. Home-based care evolution in chronic respiratory failure between 2001 and 2015 (Antadir Federation Observatory). Respiration 2018;96:446-54.

24 Veale D, Chailleux E, Taytard A, et al. Characteristics and survival of patients prescribed long-term oxygen therapy outside prescription guidelines. Eur Respir J 1998;12:780-4.

25 Austin MA, Wills KE, Blizzard L, et al. Effect of high flow oxygen on mortality in chronic obstructive pulmonary disease patients in prehospital setting: randomised controlled trial. BMJ 2010;341:c5462.

26 Cameron L, Pilcher J, Weatherall M, et al. The risk of serious adverse outcomes associated with hypoxaemia and hyperoxaemia in acute exacerbations of COPD. Postgrad Med J 2012;88:684-9.

27 Górecka D, Gorzelak K, Sliwiński P, et al. Effect of long-term oxygen therapy on survival in patients with chronic obstructive pulmonary disease with moderate hypoxaemia. Thorax 1997;52:674-9.

28 Long-Term Oxygen Treatment Trial Research Group, Albert RK, Au $\mathrm{DH}$, et al. A randomized trial of long-term oxygen for COPD with moderate desaturation. N Engl J Med 2016;375:1617-27.

29 Amalakanti S, Pentakota MR. Pulse oximetry overestimates oxygen saturation in COPD. Respir Care 2016;61:423-7.

30 Alexandre F, Varray A, Stephan Y. Reduced survival in COPD patients who received long-term oxygen therapy without meeting prescription criteria: a preliminary study. medRxiv 2019;19000760.

31 Fisher DJ, Copas AJ, Tierney JF, et al. A critical review of methods for the assessment of patient-level interactions in individual participant data meta-analysis of randomized trials, and guidance for practitioners. J Clin Epidemiol 2011;64:949-67.

32 Riley RD, Lambert PC, Abo-Zaid G. Meta-analysis of individual participant data: rationale, conduct, and reporting. BMJ 2010;340:c221.

33 Stewart LA, Clarke M, Rovers M, et al. Preferred reporting items for systematic review and meta-analyses of individual participant data: the PRISMA-IPD statement. JAMA 2015:313:1657-65.

34 Peterson J, Welch V, Losos M. The Newcastle-Ottawa scale (NOS) for assessing the quality of Nonrandomised studies in meta-analyses. Ottawa: Ottawa Hospital Research Institute, 2011.

35 Donnelly K, Bracchi R, Hewitt J, et al. Benzodiazepines, Z-drugs and the risk of hip fracture: a systematic review and meta-analysis. PLoS One 2017;12:e0174730.

36 Austin PC. A tutorial on multilevel survival analysis: methods, models and applications. Int Stat Rev 2017;85:185-203.

37 Koopman L, van der Heijden GJMG, Grobbee DE, et al. Comparison of methods of handling missing data in individual patient data metaanalyses: an empirical example on antibiotics in children with acute otitis media. Am J Epidemiol 2008;167:540-5. 